\title{
The complementary Poisson-Lindley class of distributions
}

\author{
Amal S. Hassan*, Salwa M. Assar, Kareem A. Ali \\ Institute of Statistical Studies and Research, Department of Mathematical Statistics, Cairo University, Egypt \\ *Corresponding author E-mail:dr_amal2@hotmail.com
}

\begin{abstract}
Copyright $\odot 2015$ Amal S. Hassan et al. This is an open access article distributed under the Creative Commons Attribution License, which permits unrestricted
\end{abstract} use, distribution, and reproduction in any medium, provided the original work is properly cited.

\begin{abstract}
This paper proposed a new general class of continuous lifetime distributions, which is a complementary to the PoissonLindley family proposed by Asgharzadeh et al. [3]. The new class is derived by compounding the maximum of a random number of independent and identically continuous distributed random variables, and Poisson-Lindley distribution. Several properties of the proposed class are discussed, including a formal proof of probability density, cumulative distribution, and reliability and hazard rate functions. The unknown parameters are estimated by the maximum likelihood method and the Fisher's information matrix elements are determined. Some sub-models of this class are investigated and studied in some details. Finally, a real data set is analyzed to illustrate the performance of new distributions.
\end{abstract}

Keywords: Poisson-Lindley Distribution; Complementary; Lifetime Distributions; Distribution of the Maximum.

\section{Introduction}

Lifetime distributions are of great importance in several applications for theoretical research and applied fields such as; insurance, medical, engineering, biological, communications and life testing. Recently, attempts have been made to define new lifetime probability distributions, that provide great flexibility in modeling data in practice. One way to generate a new lifetime distribution is compounding procedure. Firstly; Adamidis and Loukas [1] introduced a new distribution with decreasing failure rate by mixing the distribution of the minimum of a fixed number of exponential distributed random variables with geometric random variable. Similar procedure for deriving another lifetime distributions discussed by several authors; such as; Kus [11]; Thamasbi and Rezaei [17] and Chahkandi and Ganjali [6]. In the same way; a compound class of lifetime distributions with Poisson distribution is derived by Alkarni and Oraby [2]. Asgharzadeh et al. [3] obtained a new compound class of Poisson Lindley by compounding distribution of the minimum of a fixed number of any lifetime distribution with Poisson Lindley distribution.

Recently based on reliability studies, some researches have proposed a series of new distributions for the maximum of a sequence of identically independent distributed random variables, which represents the risk times of the system component. Louzada et al. [15] introduced a two-parameter lifetime distribution with increasing failure rate by compounding the distribution of the maximum of sequence of independent and identically components random variables from exponential distribution and geometric random variable. Next, Flores et al. [8] treat the distribution of a vector's with maximum components that are exponentially distributed in a random number of a power series distribution type. This type of distribution is called complementary exponential power series distribution. Likewise, Leahu et al. [12] introduced two new families of distributions named as max- Erlang power series distribution and minErlang power series distribution. They mixed minimum and maximum of a random number of independently; identically Erlang distributed random variables with power series distribution. Cordeiro and Silva [7] introduced the complementary class of extended Weibull power series distributions by using maximum distribution of extended Weibull.

In this article, a new compound class of Poisson-Lindley distribution is suggested by mixing the maximum of a fixed number of any continuous lifetime random variables with Poisson- Lindley random variable. In particular, some submodels of this class are derived and studied in some details. This paper is organized as following. In Section 2 , the new 
class of Poisson-Lindley lifetime distributions with its probability density, cumulative distribution, and reliability and hazard rate functions are introduced. In Section 3, some statistical measurements of the new class will be derived. Maximum likelihood estimators for the unknown parameters from the class of Poisson Lindley distributions are discussed in Section 4. Four special sub-models of the proposed class are investigated in Section 5. An application to a real data set is presented in Section 6. Finally, some concluding remarks are addressed in Section 7.

\section{The new class}

Following the same idea of Adamidis and Loukas [1], the new class of distributions is defined as follows. Let $X_{1}, X_{2}, \ldots, X_{w}$ are identically independent distributed (iid) random variables from a continuous probability density function $h(x ; \underline{\theta})$ on $(0, \infty)$, with some continuous unknown parameters $\left(\underline{\theta}=\theta_{1}, \theta_{2}, \ldots, \theta_{k}\right)$. Let $W$ be a zero truncated Poisson-Lindley random variable independent of $X^{\prime} \mathrm{s}$ with the following probability mass function:

$P(W=w)=\frac{\alpha^{2}}{\left(1+3 \alpha+\alpha^{2}\right)} \frac{2+\alpha+w}{(1+\alpha)^{w}}, w=\{1,2 \ldots \ldots\}, \alpha>0$.

Define, $\mathrm{X}=\max \left\{\mathrm{X}_{\mathrm{i}}\right\}_{\mathrm{i}=1}^{\mathrm{W}}$ as the Poisson-Lindley- $\mathrm{H}$ random variable. The conditional probability density function (PDF) of $\mathrm{X} \mid \mathrm{W}=\mathrm{W}$ is given by:

$f_{X \mid W=w}(x)=w h(x ; \underline{\theta}) H(x ; \underline{\theta})^{w-1}$

The joint distribution of $X$ and $W$ is obtained as the following:

$f_{X, W}(x, w ; \alpha, \underline{\theta})=\frac{\alpha^{2}}{\left(1+3 \alpha+\alpha^{2}\right)} \frac{2+\alpha+w}{(1+\alpha)^{w}} w h(x ; \underline{\theta}) H(x ; \underline{\theta})^{w-1}$.

So, the new class of the complementary Poisson-Lindley (CPL) lifetime distributions is derived as the marginal PDF of $X$ as follows:

$f(x ; \alpha, \underline{\theta})=\frac{\alpha^{2} h(x ; \underline{\theta})}{\left(1+3 \alpha+\alpha^{2}\right)(1+\alpha)} \times\left((2+\alpha) \sum_{w=1}^{\infty} w\left(\frac{H(x ; \underline{\theta})}{1+\alpha}\right)^{w-1}-\sum_{w=1}^{\infty} w^{2}\left(\frac{H(x ; \underline{\theta})}{1+\alpha}\right)^{w-1}\right)$,

After some simplifications, it reduces to:

$f(x ; \alpha, \underline{\theta})=\frac{\alpha^{2}(1+\alpha)^{2} h(x ; \underline{\theta})(3+\alpha-H(x ; \underline{\theta}))}{\left(1+3 \alpha+\alpha^{2}\right)(1+\alpha-H(x ; \underline{\theta}))^{3}}, x>0$.

Proposition 1:

The PDF of complementary Poisson-Lindley lifetime distributions can be written as a linear combination of $h_{i}(x, \underline{\theta}) s$

Proof

The PDF (2) can be expressed as;

$f(x ; \alpha, \underline{\theta})=\frac{\alpha^{2}(1+\alpha)^{2}}{\left(1+3 \alpha+\alpha^{2}\right)}\left[h_{1}(x ; \underline{\theta})+h_{2}(x ; \underline{\theta})\right] ;$

Where; $h_{i}(x ; \underline{\theta})=i h(x ; \underline{\theta})[1+\alpha-H(x ; \underline{\theta})]^{-(i+1)} ; i=1,2$. So, $f(x ; \alpha, \underline{\theta})$ is a linear combination of $h_{i}(x, \underline{\theta}) s$.

The corresponding cumulative distribution function (CDF) associated to (2) is given by:

$F(x ; \alpha, \underline{\theta})=\left(\frac{\alpha^{2}}{1+3 \alpha+\alpha^{2}}\right) \frac{H(x ; \underline{\theta})}{(1+\alpha-H(x ; \underline{\theta}))^{2}}\{1+\alpha+(2+\alpha)(1+\alpha-H(x ; \underline{\theta}))\}, x>0$.

Proposition 2:

$\mathrm{F}(\mathrm{x} ; \alpha, \underline{\theta}) \rightarrow \mathrm{H}(\mathrm{x} ; \underline{\theta})$ as $\alpha \rightarrow \infty$. So $\mathrm{H}(\mathrm{x} ; \underline{\theta})$ is the limiting case of $\mathrm{F}(\mathrm{x} ; \alpha, \underline{\theta})$.

Proof

$\lim _{\alpha \rightarrow \infty} F(x ; \alpha, \underline{\theta})=\lim _{\alpha \rightarrow \infty}\left(\frac{\alpha^{2}}{1+3 \alpha+\alpha^{2}}\right) \frac{H(x ; \underline{\theta})}{(1+\alpha-H(x ; \underline{\theta}))^{2}}\{1+\alpha+(2+\alpha)(1+\alpha-H(x ; \underline{\theta}))\}=H(x ; \underline{\theta})$. 
So $H(x ; \underline{\theta})$ is the limiting case of (3).

Furthermore, the reliability and hazard rate functions of complementary Poisson-Lindley lifetime distributions are obtained, respectively, as the following:

$R(x ; \alpha, \underline{\theta})=1-\frac{\alpha^{2} H(x ; \underline{\theta})\{1+\alpha+(2+\alpha)(1+\alpha-H(x ; \underline{\theta}))\}}{\left(1+3 \alpha+\alpha^{2}\right)(1+\alpha-H(x ; \underline{\theta}))^{2}}$,

and

$v(x ; \alpha, \underline{\theta})=\frac{\frac{\alpha^{2}(1+\alpha)^{2} h(x ; \underline{\theta})}{\left(1+3 \alpha+\alpha^{2}\right)}\left(\frac{3+\alpha-H(x ; \underline{\theta})}{(1+\alpha-H(x ; \underline{\theta}))^{3}}\right)}{1-\frac{\alpha^{2} H(x ; \underline{\theta})\{1+\alpha+(2+\alpha)(1+\alpha-H(x ; \underline{\theta}))\}}{\left(1+3 \alpha+\alpha^{2}\right)(1+\alpha-H(x ; \underline{\theta}))^{2}}}$

Now, let us denote a random variable $\mathrm{X}$ following the complementary Poisson-Lindley lifetime distributions with parameters $\underline{\theta}$ and $\alpha$ by $X \sim \operatorname{CPL}(\underline{\theta}, \alpha)$. This new class of distributions is presented as a generalization of several distributions.

\section{Moments and moment generating function}

Moments are commonly used to characterize the probability distribution or observed data set. Some of the most important features and characteristics of a distribution can be studied through moments (e.g. tendency, dispersion, skewness and kurtosis). The $r^{\text {th }}$ raw moment of $X$ about the origin can be determined from (2) as follows:

$E\left(X^{r}\right)=\frac{\alpha^{2}(1+\alpha)^{2}}{\left(1+3 \alpha+\alpha^{2}\right)} \int_{0}^{\infty} x^{r} h(x ; \underline{\theta})(3+\alpha-H(x ; \underline{\theta}))(1+\alpha-H(x ; \underline{\theta}))^{-3} d x$

Using the binomial expansion then $E\left(X^{r}\right)$ can be written as:

$E\left(X^{r}\right)=\frac{\alpha^{2}(1+\alpha)^{-1}}{\left(1+3 \alpha+\alpha^{2}\right)} \int_{0}^{\infty} x^{r} \sum_{i=0}^{\infty}\left(\begin{array}{c}i+2 \\ 2\end{array}\right)(1+\alpha)^{-i}(H(x ; \underline{\theta}))^{i} h(x ; \underline{\theta})(3+\alpha-H(x ; \underline{\theta})) d x$.

After some simplification,

$E\left(X^{r}\right)=\frac{\alpha^{2}(1+\alpha)^{-1}}{\left(1+3 \alpha+\alpha^{2}\right)} \sum_{i=0}^{\infty}\left(\begin{array}{c}i+2 \\ 2\end{array}\right)(1+\alpha)^{-i}[(3+\alpha) I(i)-I(i+1)]$

Where, $I(i)=\int_{0}^{\infty} x^{r} h(x, \underline{\theta})(H(x ; \underline{\theta}))^{i} d x$.

Hence, the moments of this class is obtained directly by substituting $r=1,2 \ldots$ in Equation (6).

Additionally, the moment generating function can be written as:

$M_{x}(t)=\sum_{r=0}^{\infty} \frac{t^{r}}{r !} E\left(X^{r}\right)$

Therefore, the moment generating function of CPL class of distributions is obtained from (6) as follows:

$$
M_{x}(t)=\frac{\alpha^{2}(1+\alpha)^{-1}}{\left(1+3 \alpha+\alpha^{2}\right)} \sum_{r=0}^{\infty} \frac{t^{r}}{r !} \sum_{i=0}^{\infty}\left(\begin{array}{c}
i+2 \\
2
\end{array}\right)(1+\alpha)^{-i}[(3+\alpha) I(i)-I(i+1)] .
$$

\section{Parameter estimation}

In this section; the maximum likelihood estimates (MLEs) of the model parameters of the complementary PoissonLindley class of distributions are determined from complete samples. In addition, an expression for the associated Fisher's information matrix is given.

Let $X_{1}, X_{2}, \ldots \ldots, X_{n}$ is a random sample from the new class of Poisson-Lindley with parameters $\alpha$ and $\underline{\theta}$. the loglikelihood function based on observed random sample of size $\mathrm{n}$ is given by: 
$\ell^{*}=2 n \ln \alpha(1+\alpha)-n \ln \left(1+3 \alpha+\alpha^{2}\right)+\sum_{i=1}^{n} \operatorname{lnh}\left(x_{i} ; \underline{\theta}\right)+\sum_{i=1}^{n} \ln \left(3+\alpha-H\left(x_{i} ; \underline{\theta}\right)\right)-3 \sum_{i=1}^{n} \ln (1+\alpha-$ $\left.H\left(x_{i} ; \underline{\theta}\right)\right)$.

The first partial derivatives for the log-likelihood equation with respect to $\alpha$ and $\underline{\theta}$ are given respectively as follows:

$\frac{\partial \ell^{*}}{\partial \alpha}=\frac{2 n(2 \alpha+1)}{\alpha(\alpha+1)}-\frac{n(3+2 \alpha)}{1+3 \alpha+\alpha^{2}}+\sum_{i=1}^{n} \frac{1}{3+\alpha-H\left(x_{i} ; \underline{\theta}\right)}-3 \sum_{i=1}^{n} \frac{1}{1+\alpha-H\left(x_{i} ; \underline{\theta}\right)}$

And

$\frac{\partial \ell^{*}}{\partial \underline{\theta}}=\sum_{i=1}^{n} \frac{\frac{\partial h\left(x_{i} ; \underline{\theta}\right)}{\partial \underline{\theta}}}{h\left(x_{i} ; \underline{\theta}\right)}-\sum_{i=1}^{n} \frac{\left(\frac{\partial H\left(x_{i} ; \underline{\theta}\right)}{\partial \underline{\theta}}\right)}{3+\alpha-H\left(x_{i} ; \underline{\theta}\right)}+3 \sum_{i=1}^{n} \frac{\left(\frac{\partial H\left(x_{i} ; \underline{\theta}\right)}{\partial \underline{\theta}}\right)}{1+\alpha-H\left(x_{i} ; \underline{\theta}\right)}$

The MLEs of the parameters; $\alpha$ and $\underline{\theta}$; can be obtained from (8) and (9) with respect to $\alpha$ and $\underline{\theta}$ respectively, by setting $\frac{\partial \ell^{*}}{\partial \widehat{\alpha}}=0$ and $\frac{\partial \ell^{*}}{\partial \underline{\widehat{\theta}}}=0$ and solving for the values of $\alpha$ and $\underline{\theta}$.

For large sample size, the maximum likelihood estimators, under appropriate regularity conditions, are consistent and asymptotically normally distributed. Tests of hypothesis and confidence intervals for the parameters can be obtained based on Fisher's information matrix. Therefore, the two sided approximate confidence limits for the maximum likelihood estimates $\hat{\alpha}$ and $\underline{\hat{\theta}}$ of population parameters $\alpha$ and $\underline{\theta}$ can be constructed, such that:

$\left(\begin{array}{l}\alpha \\ \underline{\theta}\end{array}\right)=\left(\begin{array}{l}\hat{\alpha} \\ \underline{\hat{\theta}}\end{array}\right) \pm Z_{\frac{\delta}{2}}\left(\sqrt{\operatorname{diagonal}\left(\hat{I}^{-1}\right)}\right)$

Where, $z_{\underline{\delta}}$ is the standard normal percentile at $\delta / 2, \delta$ is the significant level, $\hat{I}$ is the asymptotic Fisher information matrix which is obtained by substituting $\underline{\hat{\theta}}$ for $\underline{\theta}$ and $\hat{\alpha}$ for $\alpha$ as follows:

$\hat{I}=-\left(\begin{array}{ll}\frac{\partial^{2} \ell^{*}}{\partial \widehat{\alpha}^{2}} & \frac{\partial^{2} \ell^{*}}{\partial \widehat{\alpha} \partial \widehat{\underline{\theta}}} \\ \frac{\partial^{2} \ell^{*}}{\partial \widehat{\alpha} \partial \underline{\widehat{\theta}}} & \frac{\partial^{2} \ell^{*}}{\partial \widehat{\widehat{\theta}}^{2}}\end{array}\right)$.

The elements of the asymptotic Fisher information matrix $\hat{I}$ can be expressed as the following:

$\frac{\partial^{2} \ell^{*}}{\partial \widehat{\alpha}^{2}}=\frac{-2 n\left(2 \widehat{\alpha}^{2}+2 \widehat{\alpha}+1\right)}{(\widehat{\alpha}(\widehat{\alpha}+1))^{2}}+\frac{n\left(7+6 \widehat{\alpha}+2 \widehat{\alpha}^{2}\right)}{\left(1+3 \widehat{\alpha}+\widehat{\alpha}^{2}\right)^{2}}-\sum_{i=1}^{n} \frac{1}{\left(3+\widehat{\alpha}-H\left(x_{i} ; \underline{\widehat{\theta}}\right)\right)^{2}}+3 \sum_{i=1}^{n} \frac{1}{\left(1+\widehat{\alpha}-H\left(x_{i} ; \underline{\hat{\theta}}\right)\right)^{2}}$

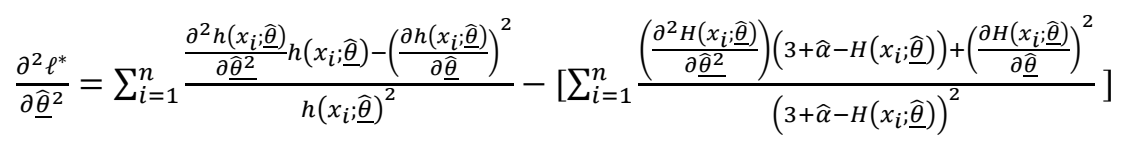

$+3 \sum_{i=1}^{n} \frac{\left(\frac{\partial^{2} H\left(x_{i} ; \underline{\hat{\theta}}\right)}{\partial \underline{\hat{\theta}}^{2}}\right)\left(1+\widehat{\alpha}-H\left(x_{i} ; \underline{\widehat{\theta}}\right)\right)+\left(\frac{\partial H\left(x_{i} ; \underline{\hat{\theta}}\right)}{\partial \underline{\underline{\theta}}}\right)^{2}}{\left(1+\widehat{\alpha}-H\left(x_{i} ; \underline{\widehat{\theta}}\right)\right)^{2}}$

$\frac{\partial^{2} \ell^{*}}{\partial \widehat{\alpha} \partial \underline{\hat{\theta}}}=\sum_{i=1}^{n} \frac{\left(\frac{\partial H\left(x_{i} ; \overrightarrow{\hat{\theta}}\right)}{\partial \underline{\hat{\theta}}}\right)}{\left(3+\widehat{\alpha}-H\left(x_{i} ; \underline{\widehat{\theta}}\right)\right)^{2}}-3 \sum_{i=1}^{n} \frac{\left(\frac{\partial H\left(x_{i} ; \underline{\hat{\theta}}\right)}{\partial \underline{\underline{\theta}}}\right)}{\left(1+\widehat{\alpha}-H\left(x_{i} ; \underline{\widehat{\theta}}\right)\right)^{2}}$.

\section{Special models}

In this section, some special cases of CPL lifetime distributions will be discussed, including the complementary Burr XII Poisson Lindley (CBXIIPL) distribution, complementary Burr III Poisson Lindley (CBIIIPL) distribution, complementary Weibull Poisson Lindley (CWPL) distribution, and complementary inverse Weibull Poisson Lindley (CIWPL) distribution. To illustrate the flexibility of distributions, plots of PDF and hazard rate function for some values of the parameter are presented. 


\subsection{Complementary Burr XII Poisson Lindley distribution}

Burr [4] introduced family of distributions which concluded twelve distributions. Burr XII distribution was the most popular between Burr distributions family. Burr [5] and Tadikamalla [16] showed that the skewness and kurtosis of Burr XII distribution have different shapes and degrees. Characteristics of Burr XII distribution are near to several distributions like exponential family, normal, lognormal...etc. To check extra properties of Burr XII distribution (see Headrick et al. [9]). The distribution function of Burr XII with shape parameters $\gamma$ and $\beta$ takes the following form:

$$
H(x ; \gamma, \beta)=1-\left(1+x^{\beta}\right)^{-\gamma}, x>0
$$

Substituting the CDF (10) and its corresponding density function into general expressions (2) and (3) to obtain the density and distribution functions of complementary Burr XII Poisson Lindley as follows:

$f(x ; \alpha, \gamma, \beta)=\left(\frac{\alpha^{2}(1+\alpha)^{2} \gamma \beta}{1+3 \alpha+\alpha^{2}}\right) x^{\beta-1}\left(1+x^{\beta}\right)^{-\gamma-1}\left(\frac{2+\alpha+\left(1+x^{\beta}\right)^{-\gamma}}{\left.\left(\alpha+\left(1+x^{\beta}\right)^{-\gamma}\right)\right)^{3}}\right), x>0, \beta, \gamma>0$,

And,

$F(x ; \alpha, \gamma, \beta)=\left(\frac{\alpha^{2}}{1+3 \alpha+\alpha^{2}}\right) \frac{\left(1-\left(1+x^{\beta}\right)^{-\gamma}\right)}{\left(\alpha+\left(1+x^{\beta}\right)^{-\gamma}\right)^{2}}\left(1+\alpha+(2+\alpha)\left(\alpha+\left(1+x^{\beta}\right)^{-\gamma}\right)\right)$

where $\alpha>0$ Is the scale parameter.

Note that; from proposition (2); as $\alpha \rightarrow \infty, F(x ; \alpha, \gamma, \beta) \rightarrow 1-\left(1+x^{\beta}\right)^{-\gamma}=H(x ; \gamma, \beta)$, which is the distribution function of Burr XII. Hence, the Burr XII is obtained as limiting distribution for CBXIIPL distribution.

The probability density function of CBXIIPL is displayed in Figure (1) for some selected values of parameter to show its flexibility to model lifetime data.

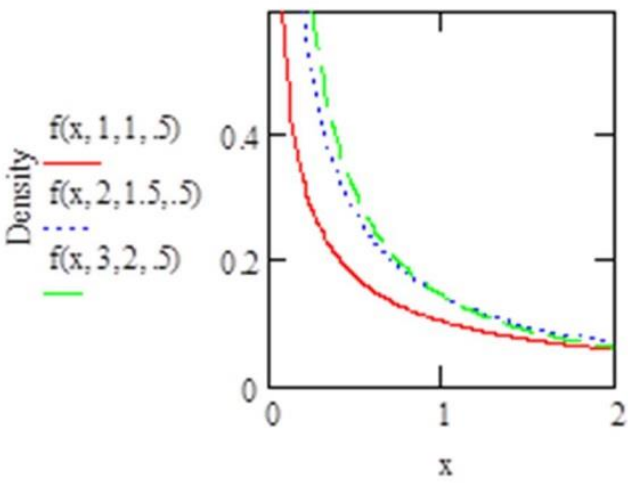

(a) $\alpha=1,2,3, \gamma=1,1.5,2, \beta=0.5$

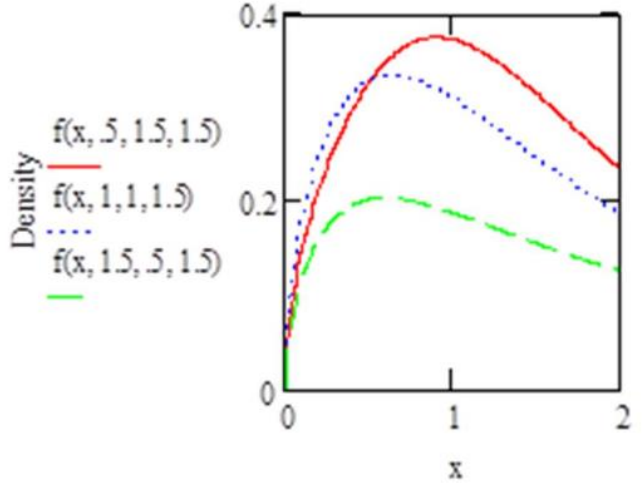

(b) $\alpha=0.5,1,1.5, \gamma=1.5,1,0.5, \beta=1.5$

Fig. 1: Plots of the CBXIIPL Densities Function for Some Parameter Values

Furthermore, from the general expressions (4) and (5), the reliability and hazard rate functions of CBXIIPL reduce to

$$
R(x ; \alpha, \gamma, \beta)=1-\frac{\alpha^{2}}{1+3 \alpha+\alpha^{2}} \frac{\left(1-\left(1+x^{\beta}\right)^{-\gamma}\right)}{\left(\alpha+\left(1+x^{\beta}\right)^{-\gamma}\right)^{2}}\left(1+\alpha+(2+\alpha)\left(\alpha+\left(1+x^{\beta}\right)^{-\gamma}\right)\right),
$$

And

$$
v(x ; \alpha, \gamma, \beta)=\frac{\frac{\alpha^{2}(1+\alpha)^{2} \gamma \beta x^{\beta-1}\left(1+x^{\beta}\right)^{-\gamma-1}}{\left(1+3 \alpha+\alpha^{2}\right)}\left(\frac{2+\alpha+\left(1+x^{\beta}\right)^{-\gamma}}{\left.\left(\alpha+\left(1+x^{\beta}\right)^{-\gamma}\right)\right)^{3}}\right)}{1-\frac{\alpha^{2}}{1+3 \alpha+\alpha^{2}} \frac{\left(1-\left(1+x^{\beta}\right)^{-\gamma}\right)}{\left(\alpha+\left(1+x^{\beta}\right)^{-\gamma}\right)^{2}}\left(1+\alpha+(2+\alpha)\left(\alpha+\left(1+x^{\beta}\right)^{-\gamma}\right)\right)} .
$$


Figure (2) represents the shapes of hazard rate function for CBXIIPL for selected values of $\alpha, \beta$ and $\gamma$. It is clear from plots that for small values of $\beta$ and $\gamma$, the hazard rate function takes decreasing form (Figure. 2(a)). Also, for large values of $\beta$ and $\gamma$, the hazard rate function takes convex form (Figure. 2(b)).

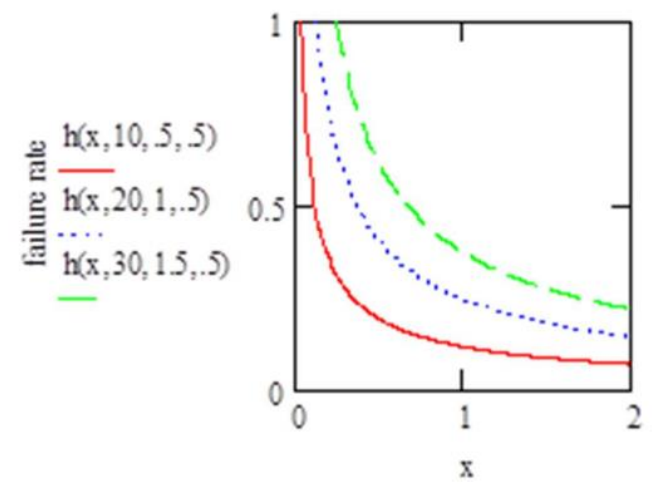

(a) $\alpha=10,20,30, \gamma=0.5,1,1.5, \beta=0.5$

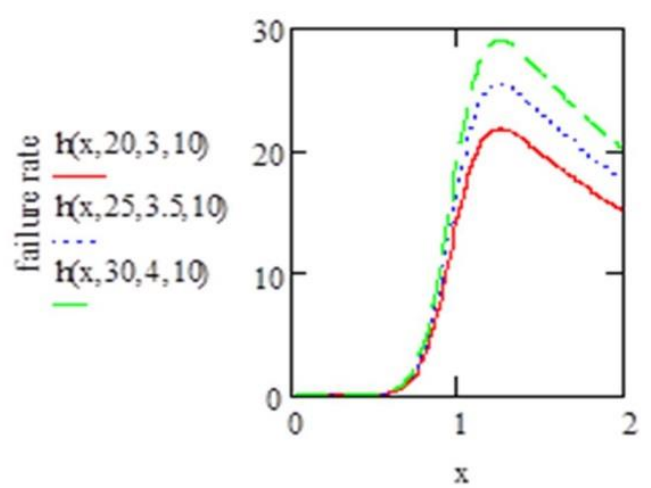

(b) $\alpha=20,25,30, \gamma=3,3.5,4, \beta=10$

Fig. 2: Plots of the CBXIIPL Hazard Rate Functions for Some Parameter Values

Based on general expression (6), the $\mathrm{r}^{\text {th }}$ raw moment of the random variable $\mathrm{X}$ about the origin having the CBXIIPL is determined as follows:

$E\left(X^{r}\right)=\frac{\alpha^{2}(1+\alpha)^{-1}}{\left(1+3 \alpha+\alpha^{2}\right)} \sum_{i=0}^{\infty}\left(\begin{array}{c}i+2 \\ 2\end{array}\right)(1+\alpha)^{-i}[(3+\alpha) I(i)-I(i+1)]$

Where, $I(i)$ is obtained by substituting (10) and its corresponding density function in (6) as follows:

$$
I(i)=\beta \gamma \int_{0}^{\infty} x^{r+\beta-1}\left(1+x^{\beta}\right)^{-\gamma}\left(1-\left(1+x^{\beta}\right)^{-\gamma}\right)^{i} d x
$$

By using binomial expansion and after some calculations, $I(i)$ takes the following form:

$$
I(i)=\sum_{k=0}^{i}\left(\begin{array}{l}
i \\
k
\end{array}\right)(-1)^{k} \frac{1}{(k+1)} \frac{1}{\Gamma(\gamma(k+1))} \Gamma\left(1+\frac{r}{\beta}\right) \Gamma\left(\gamma(k+1)-\frac{r}{\beta}\right) .
$$

Also, the moment generating function is:

$M_{x}(t)=\frac{\alpha^{2}(1+\alpha)^{-1}}{\left(1+3 \alpha+\alpha^{2}\right)} \sum_{r=0}^{\infty} \sum_{i=0}^{\infty} \frac{t^{r}}{r !}\left(\begin{array}{c}i+2 \\ 2\end{array}\right)(1+\alpha)^{-i}[(3+\alpha) I(i)-I(i+1)]$.

Furthermore, the log-likelihood function based on observed random sample of size $\mathrm{n}$ is given as follows:

$\ell^{*}=2 n \ln \alpha(1+\alpha)-n \ln \left(1+3 \alpha+\alpha^{2}\right)+n \ln \gamma \beta+(\beta-1) \sum_{i=1}^{n} \ln x_{i}$

$-(\gamma+1) \sum_{i=1}^{n} \ln \left(1+x_{i}^{\beta}\right)+\sum_{i=1}^{n} \ln \left(2+\alpha+\left(1+x_{i}^{\beta}\right)^{-\gamma}\right)-3 \sum_{i=1}^{n} \ln \left(\alpha+\left(1+x_{i}^{\beta}\right)^{-\gamma}\right)$.

The first partial derivatives for the $\log$-likelihood equation with respect to $\alpha, \gamma$ and $\beta$ are given respectively as follows:

$$
\begin{aligned}
& \frac{\partial \ell^{*}}{\partial \alpha}=\frac{2 n(2 \alpha+1)}{\alpha(\alpha+1)}-\frac{n(3+2 \alpha)}{1+3 \alpha+\alpha^{2}}+\sum_{i=1}^{n}\left[\frac{1}{2+\alpha+\left(1+x_{i}^{\beta}\right)^{-\gamma}}-\frac{3}{\alpha+\left(1+x_{i}^{\beta}\right)^{-\gamma}}\right], \\
& \frac{\partial \ell^{*}}{\partial \gamma}=\frac{n}{\gamma}-\sum_{i=1}^{n} \ln \left(1+x_{i}^{\beta}\right)-\sum_{i=1}^{n}\left[\frac{\left(1+x_{i}^{\beta}\right)^{-\gamma} \ln \left(1+x_{i}^{\beta}\right)}{2+\alpha+\left(1+x_{i}^{\beta}\right)^{-\gamma}}-\frac{3\left(1+x_{i}^{\beta}\right)^{-\gamma} \ln \left(1+x_{i}^{\beta}\right)}{\alpha+\left(1+x_{i}^{\beta}\right)^{-\gamma}}\right],
\end{aligned}
$$

And

$\frac{\partial \ell^{*}}{\partial \beta}=\frac{n}{\beta}+\sum_{i=1}^{n} \ln x_{i}-(\gamma+1) \sum_{i=1}^{n} \frac{x_{i}^{\beta} \ln x_{i}}{\left(1+x_{i}^{\beta}\right)}-\gamma \sum_{i=1}^{n}\left[\frac{\left(1+x_{i}^{\beta}\right)^{-\gamma-1} x_{i}^{\beta} \ln x_{i}}{2+\alpha+\left(1+x_{i} \beta\right)^{-\gamma}}-\frac{3\left(1+x_{i}^{\beta}\right)^{-\gamma-1} x_{i}^{\beta} \ln x_{i}}{\alpha+\left(1+x_{i}^{\beta}\right)^{-\gamma}}\right]$ 
MLEs of the unknown parameters are obtained after setting non-linear Equations (13)-(15) to be zero; $\frac{\partial \ell^{*}}{\partial \widehat{\alpha}}=0, \frac{\partial \ell^{*}}{\partial \widehat{\gamma}}=$ 0 and $\frac{\partial \ell^{*}}{\partial \widehat{\beta}}=0$. as it seems, there is no closed solution, so an extensive numerical solution will be applied.

\subsection{Complementary Burr III Poisson Lindley distribution}

The Burr III distribution properly approximates many familiar distributions such as normal, lognormal, gamma, Weibull, and exponential distributions. It plays an important role in reliability engineering, statistical quality control, and risk analysis models. Burr type III has been introduced to forestry by Lindsay et al. [14].This distribution is an important because this is inherently more flexible than the Weibull distribution, which is often used in forestry application. Burr type III covers a much larger area of the skewness-kurtosis plane than the Weibull distribution. The cumulative distribution function for Burr III distribution with shape parameters $\gamma$ and $\beta$ is given by:

$H(x ; \gamma, \beta)=\left(1+x^{-\beta}\right)^{-\gamma}, x>0$

The PDF and CDF of CBIIIPL are obtained by direct substitution of CDF (16) and its PDF in general expression (2) and (3) as the following:

$f(x ; \alpha, \gamma, \beta)=\frac{\alpha^{2}(1+\alpha)^{2} \gamma \beta x^{-\beta-1}\left(1+x^{-\beta}\right)^{-\gamma-1}}{\left(1+3 \alpha+\alpha^{2}\right)}\left(\frac{3+\alpha-\left(1+x^{-\beta}\right)^{-\gamma}}{\left.\left(1+\alpha-\left(1+x^{-\beta}\right)^{-\gamma}\right)\right)^{3}}\right), x>0, \beta, \gamma, \alpha>0$,

And

$F(x ; \alpha, \gamma, \beta)=\frac{\alpha^{2}}{1+3 \alpha+\alpha^{2}} \frac{\left(1+x^{-\beta}\right)^{-\gamma}}{\left(1+\alpha-\left(1+x^{-\beta}\right)^{-\gamma}\right)^{2}}\left(1+\alpha+(2+\alpha)\left(1+\alpha-\left(1+x^{-\beta}\right)^{-\gamma}\right)\right)$

From proposition (2); it is quite clear that as $\alpha \rightarrow \infty$, the Burr III is the limiting distribution for CBIIIPL. That is,

$\lim _{\alpha \rightarrow \infty} F(x ; \alpha, \gamma, \beta) \rightarrow\left(1+x^{-\beta}\right)^{-\gamma}=H(x ; \gamma, \beta)$

The probability density function of CBIIIPL is illustrated in Figure (3) for some selected values of parameter.

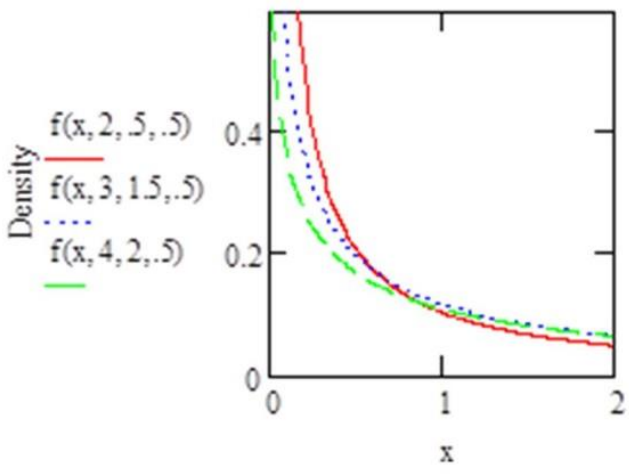

(a) $\alpha=2,3,4, \gamma=0.5,1.5,2, \beta=0.5$

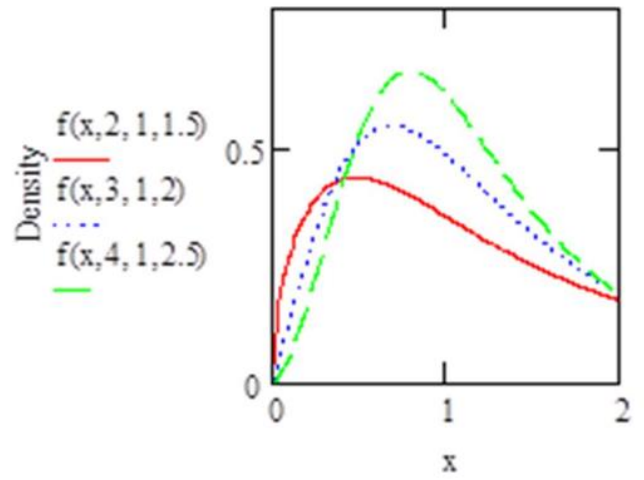

(b) $\alpha=2,3,4, \gamma=1, \beta=1.5,2,2.5$

Fig. 3: Plots of CBIIIPL Densities Function for Some Parameter Values

Furthermore, the reliability and hazard rate functions of CBIIIPL are obtained from general expressions (4) and (5) as follows:

$R(x ; \alpha, \gamma, \beta)=1-\frac{\alpha^{2}}{1+3 \alpha+\alpha^{2}} \frac{\left(1+x^{-\beta}\right)^{-\gamma}}{\left(1+\alpha-\left(1+x^{-\beta}\right)^{-\gamma}\right)^{2}}\left(1+\alpha+(2+\alpha)\left(1+\alpha-\left(1+x^{-\beta}\right)^{-\gamma}\right)\right)$

And 
$v(x ; \alpha, \gamma, \beta)=\frac{\frac{\alpha^{2}(1+\alpha)^{2} \gamma \beta x^{-\beta-1}\left(1+x^{-\beta}\right)^{-\gamma-1}}{\left(1+3 \alpha+\alpha^{2}\right)}\left(\frac{3+\alpha-\left(1+x^{-\beta}\right)^{-\gamma}}{\left.\left(1+\alpha-\left(1+x^{-\beta}\right)^{-\gamma}\right)\right)^{3}}\right)}{1-\frac{\alpha^{2}}{1+3 \alpha+\alpha^{2}\left(1+\alpha-\left(1+x^{-\beta}\right)^{-\gamma}\right)^{-\gamma}}\left(1+\alpha+(2+\alpha)\left(1+\alpha-\left(1+x^{-\beta}\right)^{-\gamma}\right)\right)}$

Figure (4) represents the shapes of hazard rate function for CBIIIPL for selected values of $\alpha, \beta$ and $\gamma$. Clearly, for small values of $\beta$ and $\gamma$, plots of the hazard rate function take decreasing form (Figure. 4(a)). Also, the hazard rate function takes convex form for large values of $\beta$ and $\gamma$ ((Figure. 4(b)).

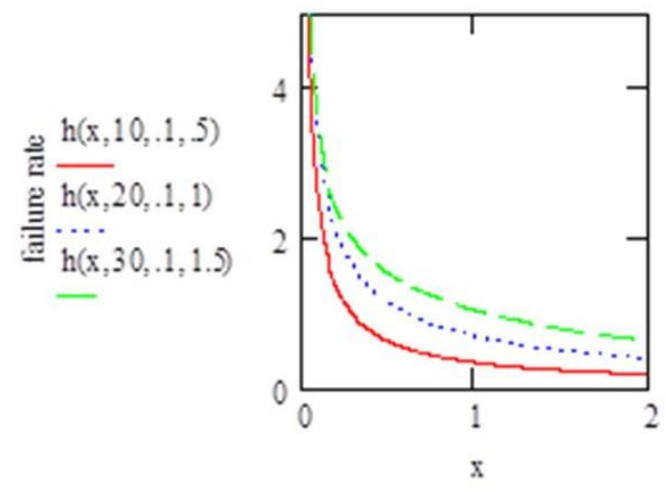

(a) $\alpha=10,20,30, \gamma=0.1, \beta=0.5,1,1.5$

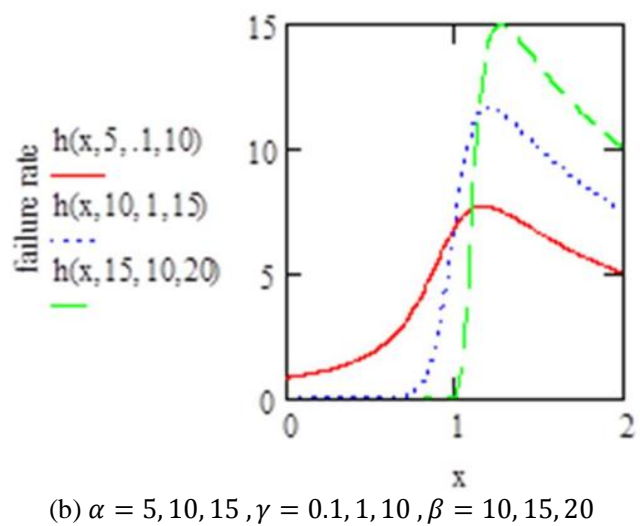

(b) $\alpha=5,10,15, \gamma=0.1,1,10, \beta=10,15,20$

Fig. 4: Plots of the CBIIIPL Hazard Rate Functions for Some Parameter Values

To obtain the $\mathrm{r}^{\text {th }}$ raw moment of CBIIIPL, firstly it must to obtain the integrated part, I(i), defined in (6) as follows:

$$
I(i)=\int_{0}^{\infty} \beta \gamma x^{r-\beta-1}\left(1+x^{\beta}\right)^{-\gamma(i+1)} d x=\frac{\Gamma\left(1-\frac{r}{\beta}\right) \Gamma\left(\gamma(i+1)+\frac{r}{\beta}\right)}{(i+1) \Gamma \gamma(i+1)}
$$

After substituting (19) in (6), the $\mathrm{r}^{\text {th }}$ raw moment of CBIIIPL distribution takes the following form:

$E\left(X^{r}\right)=\frac{\alpha^{2}(1+\alpha)^{-1}}{\left(1+3 \alpha+\alpha^{2}\right)} \sum_{i=0}^{\infty}\left(\begin{array}{c}i+2 \\ 2\end{array}\right)(1+\alpha)^{-i} \Gamma\left(1-\frac{r}{\beta}\right)\left\{\frac{(3+\alpha) \Gamma\left(\gamma(i+1)+\frac{r}{\beta}\right)}{(i+1) \Gamma(\gamma(i+1))}-\frac{\Gamma\left(\gamma(i+2)+\frac{r}{\beta}\right)}{(i+2) \Gamma(\gamma(i+2))}\right\}$.

Likewise, the moment generating function can be expressed as the following:

$M_{x}(t)=\sum_{r=0}^{\infty} \sum_{i=0}^{\infty} \frac{t^{r}}{r !} \frac{\alpha^{2}(1+\alpha)^{-1}}{\left(1+3 \alpha+\alpha^{2}\right)}\left(\begin{array}{c}i+2 \\ 2\end{array}\right)(1+\alpha)^{-i} \Gamma\left(1-\frac{r}{\beta}\right)\left\{\frac{(3+\alpha) \Gamma\left(\gamma(i+1)+\frac{r}{\beta}\right)}{(i+1) \Gamma(\gamma(i+1))}-\frac{\Gamma\left(\gamma(i+2)+\frac{r}{\beta}\right)}{(i+2) \Gamma(\gamma(i+2))}\right\}$.

The log-likelihood function based on the observed sample of size $\mathrm{n}$ from CBIIIPL distribution is given by:

$$
\begin{aligned}
& \ell^{*}=2 n \ln \alpha(1+\alpha)-n \ln \left(1+3 \alpha+\alpha^{2}\right)+n \ln \beta \gamma-(\beta+1) \sum_{i=1}^{n} \ln x_{i}-(\gamma+1) \sum_{i=1}^{n} \ln \left(1+x_{i}^{-\beta}\right) \\
& +\sum_{i=1}^{n} \ln \left(3+\alpha-\left(1+x_{i}^{-\beta}\right)^{-\gamma}\right)-3 \sum_{i=1}^{n} \ln \left(1+\alpha-\left(1+x_{i}^{-\beta}\right)^{-\gamma}\right) .
\end{aligned}
$$

The first partial derivatives for the log-likelihood equation with respect to $\alpha, \gamma$ and $\beta$ are given respectively as follows:

$\frac{\partial \ell^{*}}{\partial \alpha}=\frac{2 n(2 \alpha+1)}{\alpha(\alpha+1)}-\frac{n(3+2 \alpha)}{1+3 \alpha+\alpha^{2}}+\sum_{i=1}^{n} \frac{1}{3+\alpha-\left(1+x_{i}^{-\beta}\right)^{-\gamma}}-\sum_{i=1}^{n} \frac{3}{1+\alpha-\left(1+x_{i}^{-\beta}\right)^{-\gamma}}$.

$\frac{\partial \ell^{*}}{\partial \gamma}=\frac{n}{\gamma}-\sum_{i=1}^{n} \ln \left(1+x_{i}^{\beta}\right)+\sum_{i=1}^{n} \frac{\left(1+x_{i}^{-\beta}\right)^{-\gamma} \ln \left(1+x_{i}^{-\beta}\right)}{3+\alpha-\left(1+x_{i}^{-\beta}\right)^{-\gamma}}-3 \sum_{i=1}^{n} \frac{\left(1+x_{i}^{-\beta}\right)^{-\gamma} \ln \left(1+x_{i}^{-\beta}\right)}{1+\alpha-\left(1+x_{i}^{-\beta}\right)^{-\gamma}}$.

And

$\frac{\partial \ell^{*}}{\partial \beta}=\frac{n}{\beta}-\sum_{i=1}^{n} \ln x_{i}+(\gamma+1) \sum_{i=1}^{n} \frac{x_{i}^{-\beta} \ln x_{i}}{1+x_{i}^{-\beta}}-\gamma \sum_{i=1}^{n} \frac{\left(1+x_{i}^{-\beta}\right)^{-\gamma-1} x_{i}^{-\beta} \ln x_{i}}{3+\alpha-\left(1+x_{i}^{-\beta}\right)^{-\gamma}}$ 
$+3 \gamma \sum_{i=1}^{n} \frac{\left(1+x_{i}^{-\beta}\right)^{-\gamma-1} x_{i}^{-\beta} \ln x_{i}}{1+\alpha-\left(1+x_{i}^{-\beta}\right)^{-\gamma}}$

To achieve estimations via maximum likelihood method, it is not easy to solve equations $\frac{\partial \ell^{*}}{\partial \widehat{\alpha}}=0, \frac{\partial \ell^{*}}{\partial \widehat{\gamma}}=0$ and $\frac{\partial \ell^{*}}{\partial \widehat{\beta}}=0$, directly, so an iterative technique will be used.

\subsection{Complementary Weibull Poisson Lindley distribution}

The Weibull distribution is an important for modeling and lifetime data analysis in biological, medical and engineering sciences. It can therefore model a great variety of data and life characteristics. It is used extensively in reliability applications to model failure times. The distribution function of Weibull distribution with the shape parameter $\beta$ and scale parameter $\lambda$ takes the following form:

$H(x ; \lambda, \beta)=1-e^{-\lambda(x)^{\beta}}, x>0$ and $\beta, \lambda>0$.

The probability density and distribution functions of CWPL are determined from general expression (2) and (3) by direct substitution of CDF (23) and its corresponding density function as the following:

$f(x ; \alpha, \lambda, \beta)=\frac{\alpha^{2}(1+\alpha)^{2} \beta \lambda}{\left(1+3 \alpha+\alpha^{2}\right)} x^{\beta-1} e^{-\lambda(x)^{\beta}} \frac{\left(2+\alpha+e^{-\lambda(x)^{\beta}}\right)}{\left(\alpha+e^{-\lambda(x)^{\beta}}\right)^{3}}, x>0$,

And

$F(x ; \alpha, \lambda, \beta)=\left(\frac{\alpha^{2}}{1+3 \alpha+\alpha^{2}}\right) \frac{\left(1-e^{-\lambda(x)^{\beta}}\right)}{\left(\alpha+e^{-\lambda(x)^{\beta}}\right)^{2}}\left(1+\alpha+(2+\alpha)\left(\alpha+e^{-\lambda(x)^{\beta}}\right)\right)$.

Clearly, from proposition $(2) ; \lim _{\alpha \rightarrow \infty} F(x ; \alpha, \lambda, \beta) \rightarrow 1-e^{-\lambda(x)^{\beta}}=H(x ; \lambda, \beta)$, that is; the Weibull distribution is the limiting case when $\alpha \rightarrow \infty$.

The probability density function of CWPL is displayed in Figure (5) for some selected values of parameter

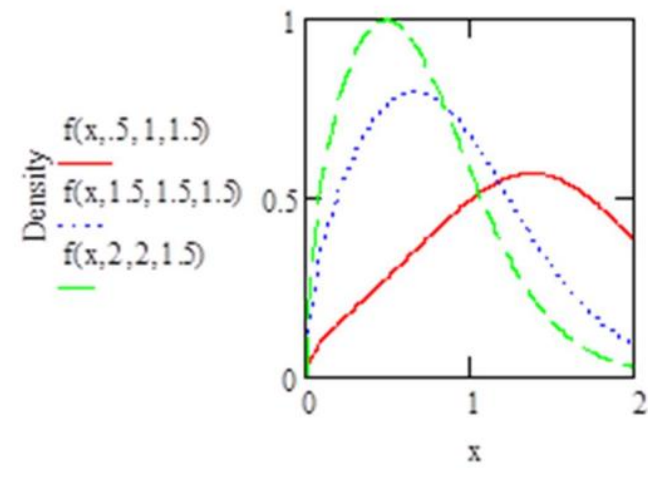

(a) $\alpha=0.5,1.5,2, \lambda=1,1.5,2, \beta=1.5$

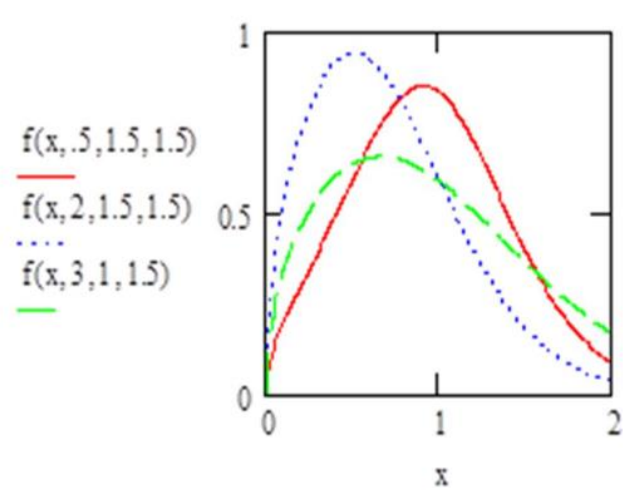

(b) $\alpha=0.5,2,3, \lambda=1.5,1, \beta=1.5$

Fig. 5: Plots of CWPL Densities Function for Some Parameter Values

Also, the reliability and hazard rate functions of CWPL are as follows:

$R(x ; \alpha, \lambda, \beta)=1-\frac{\alpha^{2}}{1+3 \alpha+\alpha^{2}} \frac{\left(1-e^{-\lambda(x)^{\beta}}\right)}{\left(\alpha+e^{-\lambda(x)^{\beta}}\right)^{2}}\left(1+\alpha+(2+\alpha)\left(\alpha+e^{-\lambda(x)^{\beta}}\right)\right)$,

And 


$$
v(x ; \alpha, \lambda, \beta)=\frac{\frac{\alpha^{2}(1+\alpha)^{2} \beta \lambda x^{\beta-1} e^{-\lambda(x)^{\beta}}\left(2+\alpha+e^{\left.-\lambda(x)^{\beta}\right)}\right.}{\left(1+3 \alpha+\alpha^{2}\right)}\left(\alpha+e^{\left.\left.-\lambda(x)^{\beta}\right)\right)^{3}}\right.}{1-\frac{\alpha^{2}\left(1-e^{-\lambda(x)^{\beta}}\right)}{\left(1+3 \alpha+\alpha^{2}\right)\left(\alpha+e^{-\lambda(x)^{\beta}}\right)^{2}}\left(1+\alpha+(2+\alpha)\left(\alpha+e^{-\lambda(x) \beta}\right)\right)} .
$$

Figure (6) shows the shapes of hazard rate functions for CWPL for some selected value of $\alpha, \beta$ and $\lambda$. Clearly, the plots of the hazard rate function take decreasing form for the same value of $\beta$ and as the values of $\lambda$ increase (see Figure $6(\mathrm{a})$ ). The hazard rate function takes increasing form for large values of $\beta$ and $\lambda$ (see Figure. 6(b)).

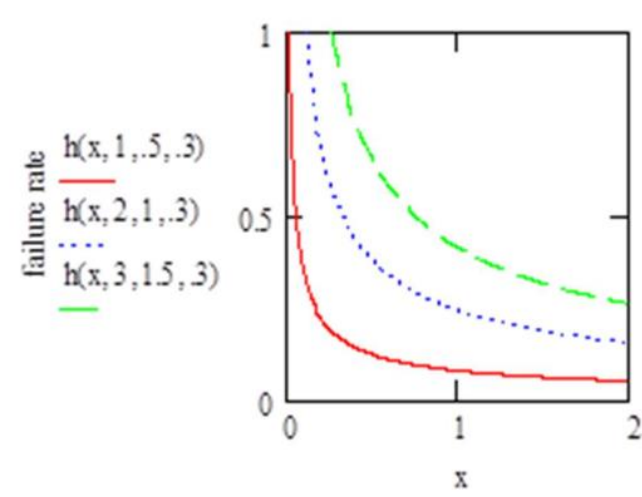

(a) $\alpha=1,2,3, \lambda=0.5,1,1.5, \beta=0.3$

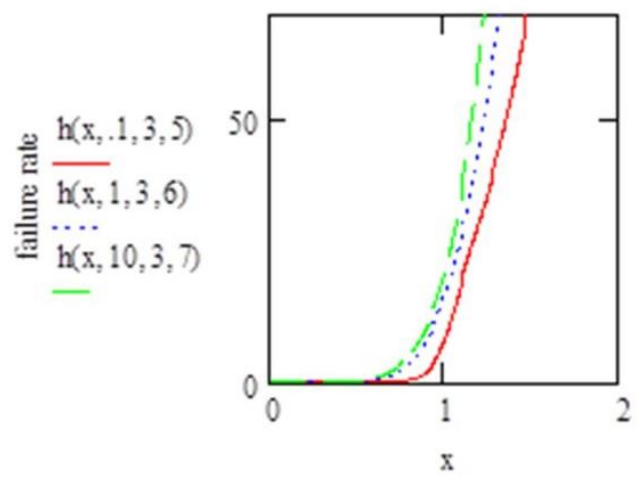

(b) $\alpha=0.1,1,10, \lambda=3, \beta=5,6,7$

Fig. 6: Plots of the CWPL Hazard Rate Functions for Some Parameter Values

The $\mathrm{r}^{\text {th }}$ raw moments and moment generating function of CWPL distribution are obtained, based on general expressions (6) and (7), as follows:

$E\left(X^{r}\right)=\frac{\alpha^{2}(1+\alpha)^{-1}}{\left(1+3 \alpha+\alpha^{2}\right)} \sum_{i=0}^{\infty}\left(\begin{array}{c}i+2 \\ 2\end{array}\right)(1+\alpha)^{-i}[(3+\alpha) I(i)-I(i+1)]$

And,

$M_{x}(t)=\frac{\alpha^{2}(1+\alpha)^{-1}}{\left(1+3 \alpha+\alpha^{2}\right)} \sum_{r=0}^{\infty} \sum_{i=0}^{\infty} \frac{t^{r}}{r !}\left(\begin{array}{c}i+2 \\ 2\end{array}\right)(1+\alpha)^{-i}[(3+\alpha) I(i)-I(i+1)]$

Where,

$I(i)=\int_{0}^{\infty} \beta \lambda x^{r+\beta-1} e^{-\lambda(x)^{\beta}}\left(1-e^{-\lambda(x)^{\beta}}\right)^{i} d x=\sum_{k=0}^{i}\left(\begin{array}{l}i \\ k\end{array}\right)(-1)^{k} \frac{1}{(k+1)} \frac{1}{(\lambda(k+1))^{\frac{r}{\beta}}} \Gamma\left(1+\frac{r}{\beta}\right)$

The log-likelihood function based on observed random sample of size $\mathrm{n}$ for CWPL distribution is given by:

$\ell^{*}=2 n \ln \alpha(1+\alpha)-n \ln \left(1+3 \alpha+\alpha^{2}\right)+n \ln \lambda \beta+(\beta-1) \sum_{i=1}^{n} \ln x_{i}-\lambda \sum_{i=1}^{n}\left(x_{i}\right)^{\beta}$

$+\sum_{i=1}^{n} \ln \left(2+\alpha+e^{-\lambda\left(x_{i}\right)^{\beta}}\right)-3 \sum_{i=1}^{n} \ln \left(\alpha+e^{-\lambda\left(x_{i}\right)^{\beta}}\right)$

The first partial derivatives for the log-likelihood equation with respect to $\alpha, \lambda$ and $\beta$ are given respectively as follows;

$\frac{\partial \ell^{*}}{\partial \alpha}=\frac{2 n(2 \alpha+1)}{\alpha(\alpha+1)}-\frac{n(3+2 \alpha)}{1+3 \alpha+\alpha^{2}}+\sum_{i=1}^{n}\left[\frac{1}{2+\alpha+e^{-\lambda\left(x_{i}\right)^{\beta}}}-\frac{3}{\alpha+e^{-\lambda\left(x_{i}\right)^{\beta}}}\right]$,

$\frac{\partial e^{*}}{\partial \lambda}=\frac{n}{\lambda}-\sum_{i=1}^{n}\left(x_{i}\right)^{\beta}-\sum_{i=1}^{n}\left[\frac{e^{-\lambda\left(x_{i}\right)^{\beta}}\left(x_{i}\right)^{\beta}}{2+\alpha+e^{-\lambda\left(x_{i}\right)^{\beta}}}-\frac{3 e^{-\lambda\left(x_{i}\right)^{\beta}}\left(x_{i}\right)^{\beta}}{\alpha+e^{-\lambda\left(x_{i}\right)^{\beta}}}\right]$,

And 
$\frac{\partial e^{*}}{\partial \beta}=\frac{n}{\beta}+\sum_{i=1}^{n} \ln x_{i}-\lambda \sum_{i=1}^{n}\left(x_{i}\right)^{\beta} \ln \left(x_{i}\right)-\lambda \sum_{i=1}^{n} \frac{\left(x_{i}\right)^{\beta} \ln \left(x_{i}\right) e^{-\lambda\left(x_{i}\right)^{\beta}}}{2+\alpha+e^{-\lambda\left(x_{i}\right)^{\beta}}}+3 \lambda \sum_{i=1}^{n} \frac{\left(x_{i}\right)^{\beta} \ln \left(x_{i}\right) e^{-\lambda\left(x_{i}\right)^{\beta}}}{\alpha+e^{-\lambda\left(x_{i}\right)^{\beta}}}$.

The MLEs of the parameters $\alpha, \lambda$ and $\beta$ are obtained numerically by solving a system of nonlinear Equations (26) to (28), after setting with zero as $\frac{\partial \ell^{*}}{\partial \widehat{\alpha}}=0, \frac{\partial \ell^{*}}{\partial \widehat{\lambda}}=0$ and $\frac{\partial \ell^{*}}{\partial \widehat{\beta}}=0$. As it seems, there is no closed solution, so an extensive numerical solution will be applied.

\subsection{Complementary inverse Weibull Poisson Lindley distribution}

The inverse Weibull distribution is another life time probability distribution which can be used in the reliability engineering discipline. The inverse Weibull distribution can be used to model a variety of failure characteristics such as infant mortality, useful life and wear-out periods (see Khan et al. [10]). The cumulative distribution function for inverse Weibull distribution with shape parameter $\beta$ and scale parameter $\lambda$ takes the following form:

$H(x ; \lambda, \beta)=e^{-\lambda(x)^{-\beta}}, x>0, \beta$ and $\lambda>0$.

The PDF of CIWPL is obtained by direct substitution of CDF (29) and its PDF in PDF (2) and CDF (3) as the following:

$f(x ; \alpha, \lambda, \beta)=\frac{\alpha^{2}(1+\alpha)^{2} \beta \lambda x^{-\beta-1} e^{-\lambda(x)^{-\beta}}}{\left(1+3 \alpha+\alpha^{2}\right)} \frac{3+\alpha-e^{-\lambda(x)^{-\beta}}}{\left.\left(1+\alpha-e^{-\lambda(x)^{-\beta}}\right)\right)^{3}}, x>0, \alpha, \beta, \gamma>0$,

And

$F(x ; \alpha, \lambda, \beta)=\frac{\alpha^{2}}{1+3 \alpha+\alpha^{2}} \frac{\left(e^{-\lambda(x)^{-\beta}}\right)}{\left(1+\alpha-e^{-\lambda(x)^{-\beta}}\right)^{2}}\left(1+\alpha+(2+\alpha)\left(1+\alpha-e^{-\lambda(x)^{-\beta}}\right)\right)$.

Note that: as $\alpha \rightarrow \infty$, the inverse Weibull distribution is obtained as the limiting distribution for CIWPL, i.e $\lim _{\alpha \rightarrow \infty} F(x ; \alpha, \lambda, \beta) \rightarrow e^{-\lambda(x)^{-\beta}}=H(x ; \lambda, \beta)$ which is the distribution function of inverse Weibull distribution.

Figure (7) represents the PDF of CIWPL distribution for some selected values of parameter

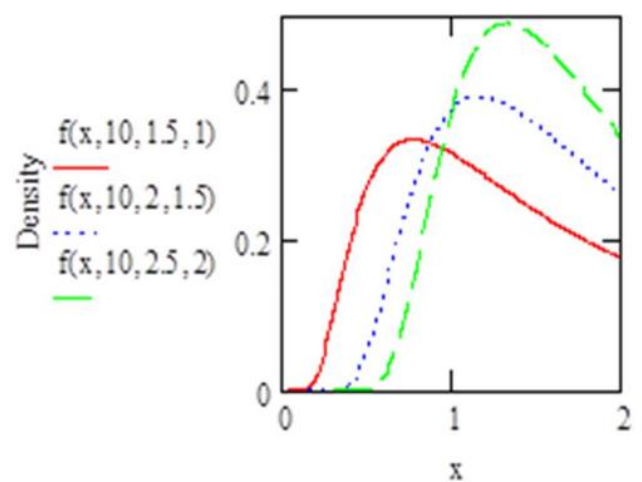

(a) $\alpha=10, \lambda=1.5,2,2.5, \beta=1,1.5,2$

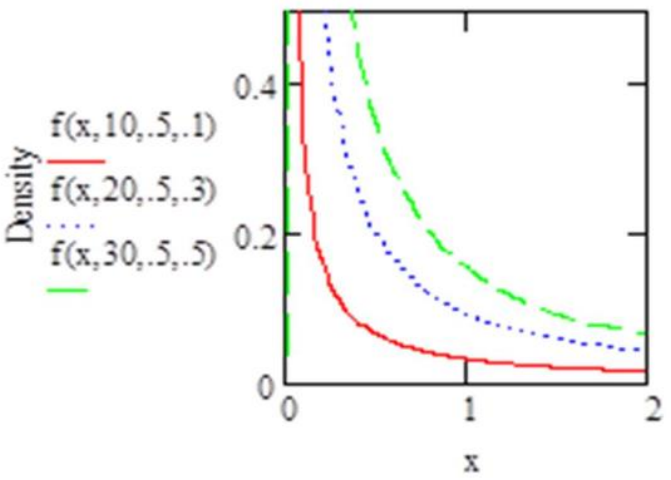

(b) $\alpha=10,20,30, \lambda=0.5, \beta=0.1,0.3,0.5$

Fig. 7: Plots of CIWPL Densities Function for Some Parameter Values

In addition, the reliability and hazard rate functions of CIWPL are given, respectively, as follows:

$$
R(x ; \alpha, \lambda, \beta)=1-\frac{\alpha^{2}}{1+3 \alpha+\alpha^{2}} \frac{e^{-\lambda(x)^{-\beta}}}{\left(1+\alpha-e^{-\lambda(x)^{-\beta}}\right)^{2}}\left(1+\alpha+(2+\alpha)\left(1+\alpha-e^{-\lambda(x)^{-\beta}}\right)\right),
$$

And

$$
v(x ; \alpha, \lambda, \beta)=\frac{\frac{\alpha^{2}(1+\alpha)^{2} \beta \lambda x^{-\beta-1} e^{-\lambda(x)^{-\beta}}\left(3+\alpha-e^{-\lambda(x)^{-\beta}}\right.}{\left(1+3 \alpha+\alpha^{2}\right)}\left(1+\alpha-e^{\left.\left.-\lambda(x)^{-\beta}\right)\right)^{3}}\right.}{1-\frac{\alpha^{2}}{1+3 \alpha+\alpha^{2}} \frac{\left(e^{-\lambda(x)^{-\beta}}\right)}{\left(1+\alpha-e^{-\lambda(x)^{-\beta}}\right)^{2}}\left(1+\alpha+(2+\alpha)\left(1+\alpha-e^{-\lambda(x)^{-\beta}}\right)\right)} .
$$


Figure (8) represents the hazard rate function for CIWPL for some selected values of $\alpha, \beta$ and $\lambda$. It is quite clear from plots that for small values of $\beta$ and $\lambda$ the hazard rate function takes decreasing form (see Figure. 8(a)). Also, for large values of $\beta$ and $\lambda$ the hazard rate function takes convex form (see Figure. 8(b)).

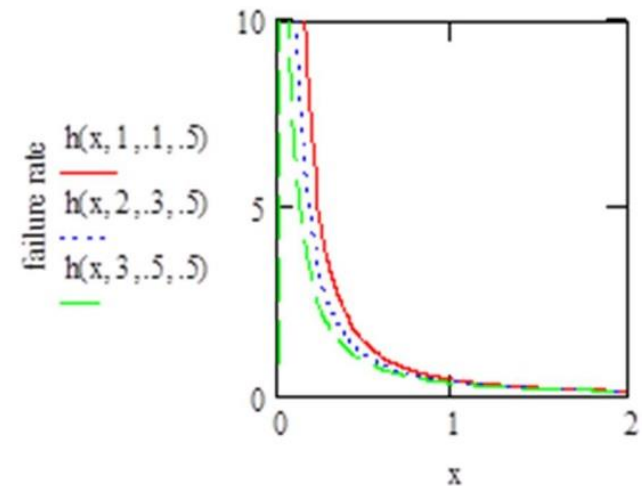

(a) $\alpha=1,2,3, \lambda=0.1,0.3,0.5, \beta=0.5$

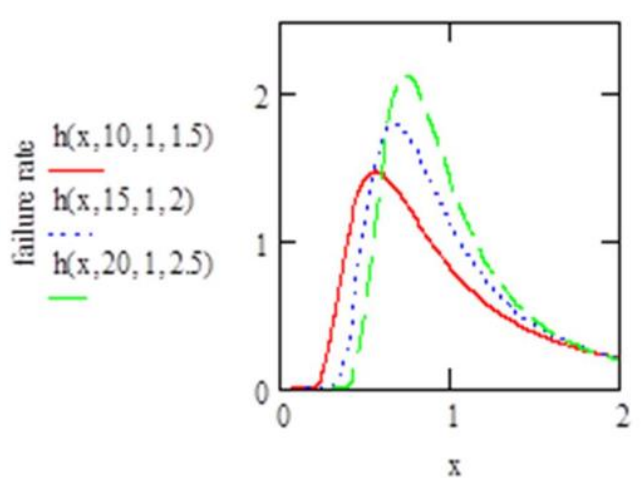

(b) $\alpha=10,15,20, \lambda=1, \beta=1.5,2,2.5$

Fig. 8: Plots of the CIWPL Hazard Rate Functions for Some Parameter Values

Standard calculations show that the $\mathrm{r}^{\text {th }}$ moment population and moment generating function of CIWPL distribution are:

$$
E\left(X^{r}\right)=\frac{\alpha^{2}(1+\alpha)^{-1}}{\left(1+3 \alpha+\alpha^{2}\right)} \sum_{i=0}^{\infty}\left(\begin{array}{c}
i+2 \\
2
\end{array}\right)(1+\alpha)^{-i} \Gamma\left(1-\frac{r}{\beta}\right) \lambda^{\frac{r}{\beta}}\left\{(3+\alpha)(i+1)^{\frac{r}{\beta}-1}-(i+2)^{\frac{r}{\beta}-1}\right\}
$$

And,

$M_{x}(t)=\frac{\alpha^{2}(1+\alpha)^{-1}}{\left(1+3 \alpha+\alpha^{2}\right)} \sum_{r=0}^{\infty} \sum_{i=0}^{\infty} \frac{t^{r}}{r !}\left(\begin{array}{c}i+2 \\ 2\end{array}\right)(1+\alpha)^{-i} \Gamma\left(1-\frac{r}{\beta}\right) \lambda^{\frac{r}{\beta}}\left\{(3+\alpha)(i+1)^{\frac{r}{\beta}-1}-(i+2)^{\frac{r}{\beta}-1}\right\}$

The log-likelihood function based on observed random sample of size $\mathrm{n}$ is given by:

$$
\begin{aligned}
& \ell^{*}=2 n \ln \alpha(1+\alpha)-n \ln \left(1+3 \alpha+\alpha^{2}\right)+n \ln \lambda \beta-(\beta+1) \sum_{i=1}^{n} \ln x_{i}-\lambda \sum_{i=1}^{n}\left(x_{i}\right)^{-\beta} \\
& +\sum_{i=1}^{n} \ln \left(3+\alpha-e^{-\lambda\left(x_{i}\right)^{-\beta}}\right)-3 \sum_{i=1}^{n} \ln \left(1+\alpha-e^{-\lambda\left(x_{i}\right)^{-\beta}}\right)
\end{aligned}
$$

The first partial derivatives for the log-likelihood equation with respect to $\alpha, \lambda$ and $\beta$ are given respectively as follows:

$$
\begin{aligned}
& \frac{\partial \ell^{*}}{\partial \alpha}=\frac{2 n(2 \alpha+1)}{\alpha(\alpha+1)}-\frac{n(3+2 \alpha)}{1+3 \alpha+\alpha^{2}}+\sum_{i=1}^{n} \frac{1}{3+\alpha-e^{-\lambda\left(x_{i}\right)^{-\beta}}}-3 \sum_{i=1}^{n} \frac{1}{1+\alpha-e^{-\lambda\left(x_{i}\right)^{-\beta}}}, \\
& \frac{\partial \ell^{*}}{\partial \lambda}=\frac{n}{\lambda}-\sum_{i=1}^{n}\left(x_{i}\right)^{-\beta}+\sum_{i=1}^{n} \frac{e^{-\lambda\left(x_{i}\right)^{-\beta}}\left(x_{i}\right)^{-\beta}}{3+\alpha-e^{-\lambda\left(x_{i}\right)^{-\beta}}}-3 \sum_{i=1}^{n} \frac{e^{-\lambda\left(x_{i}\right)^{-\beta}}\left(x_{i}\right)^{-\beta}}{1+\alpha-e^{-\lambda\left(x_{i}\right)^{-\beta}}}
\end{aligned}
$$

And

$\frac{\partial \ell^{*}}{\partial \beta}=\frac{n}{\beta}-\sum_{i=1}^{n} \ln x_{i}+\lambda \sum_{i=1}^{n}\left(x_{i}\right)^{-\beta} \ln \left(x_{i}\right)-\sum_{i=1}^{n} \frac{\lambda\left(x_{i}\right)^{-\beta} \ln \left(x_{i}\right) e^{-\lambda\left(x_{i}\right)^{-\beta}}}{3+\alpha-e^{-\lambda\left(x_{i}\right)^{-\beta}}}+3 \sum_{i=1}^{n} \lambda \frac{\left(x_{i}\right)^{-\beta} \ln \left(x_{i}\right) e^{-\lambda\left(x_{i}\right)^{-\beta}}}{1+\alpha-e^{-\lambda\left(x_{i}\right)^{-\beta}}}$.

MLEs of the $\alpha, \lambda$ and $\beta$ can be worked out by letting the partial derivatives $\frac{\partial \ell^{*}}{\partial \widehat{\alpha}}, \frac{\partial \ell^{*}}{\partial \widehat{\lambda}}$ and $\frac{\partial \ell^{*}}{\partial \widehat{\beta}}$ to be zero. It is clear that there are no exact solutions to non-linear equations, so an iterative technique will be used.

\section{Application to real data}

In this section, CBXIIPL, CBIIIPL CWPL and CIWPL models are fitted to real data. Table (1) contains a real data set corresponding to remission times (in months) of random sample of 128 bladder cancer patients which reported in Lee and Wang [13]. 
Table 1: The Remission Times (in Months) of 128 Bladder Cancer

\begin{tabular}{cccccccccc}
\hline 0.08 & 2.09 & 3.48 & 4.87 & 6.94 & 8.66 & 13.11 & 23.63 & 0.2 & 2.23 \\
0.52 & 4.98 & 6.97 & 9.02 & 13.29 & 0.4 & 2.26 & 3.57 & 5.06 & 7.09 \\
0.22 & 13.8 & 25.74 & 0.5 & 2.46 & 3.46 & 5.09 & 7.26 & 9.47 & 14.24 \\
0.82 & 0.51 & 2.54 & 3.7 & 5.17 & 7.28 & 9.74 & 14.76 & 26.31 & 0.81 \\
0.62 & 3.28 & 5.32 & 7.32 & 10.06 & 14.77 & 32.15 & 2.64 & 3.88 & 5.32 \\
0.39 & 10.34 & 14.38 & 34.26 & 0.9 & 2.69 & 4.18 & 5.34 & 7.59 & 10.66 \\
0.96 & 36.66 & 1.05 & 2.69 & 4.23 & 5.41 & 7.62 & 10.75 & 16.62 & 43.01 \\
0.19 & 2.75 & 4.26 & 5.41 & 7.63 & 17.12 & 46.12 & 1.26 & 2.83 & 4.33 \\
0.66 & 11.25 & 17.14 & 79.05 & 1.35 & 2.87 & 5.62 & 7.87 & 11.64 & 17.36 \\
0.4 & 3.02 & 4.34 & 5.71 & 7.93 & 11.79 & 18.1 & 1.46 & 4.4 & 5.85 \\
0.26 & 11.98 & 19.13 & 1.76 & 3.25 & 4.5 & 6.25 & 8.37 & 12.02 & 2.02 \\
0.31 & 4.51 & 6.54 & 8.53 & 12.03 & 20.28 & 2.02 & 3.36 & 6.76 & 12.07 \\
0.73 & 2.07 & 3.36 & 6.39 & 8.65 & 12.63 & 22.69 & 5.49 & & \\
\hline
\end{tabular}

Method of the maximum likelihood is used to get estimates of the unknown parameters and its standard errors (SE) for each model. To compare the four distributions, criteria like; Akaike information criterion (AIC), Bayesian information criterion (BIC), minus log-likelihood $\left(-\ell^{*}\right)$ and Kolmogorov-Smirnov (K-S) goodness of fit test statistics will be considered for the above data set. Convergence condition for the large samples is satisfied, so parameters values are replaced by their likelihood estimators to estimate K-S statistics.

Table (2) shows parameter MLEs (with the corresponding standard error in parentheses), values of log-likelihood, values of AIC, values of BIC and P-values based on one sample K-S statistics.

Table 2: Statistical Analysis for Bladder Cancer Patient's Data

\begin{tabular}{cccccc}
\hline Distribution & \multicolumn{1}{c}{ Estimates(SE) } & $-\ell^{*}$ & AIC & BIC & P-value \\
\hline \multirow{2}{*}{ CWPL } & $\hat{\alpha}=8.691(22.116)$ & & & & \\
& $\hat{\lambda}=0.162(0.058)$ & 402.192 & 810.383 & 681.772 & 0.00 \\
& $\hat{\beta}=0.899(0.085)$ & & & & \\
CIWPL & $\hat{\alpha}=0.079(0.044)$ & & & & \\
& $\hat{\lambda}=0.198(0.109)$ & 410.769 & 827.538 & 681.793 & 0.00 \\
& $\hat{\beta}=1.12(0.075)$ & & & \\
CBXIIPL & $\hat{\alpha}=0.106(0.073)$ & 404.442 & 814.884 & 681.768 & $0.824^{*}$ \\
& $\hat{\gamma}=2.084(0.909)$ & & & \\
CBIIIPL & $\hat{\beta}=0.779(0.273)$ & & & \\
& $\hat{\alpha}=0.197(0.101)$ & & & & \\
& $\hat{\gamma}=2.89(0.362)$ & 406.338 & 818.676 & 681.774 & $0.776^{*}$ \\
\hline
\end{tabular}

The values in Table (2) indicate that the P-values for K-S statistics have the smallest values for the data set under CBXIIPL and CBIIIPL models with regard to the other models.

The quantile-quantile or Q-Q plot is used to check the validity of the distributional assumption for the data. Figure (9) shows that the data seems CBXIIPL and CBIIIPL distributions provide the best fit to this data 


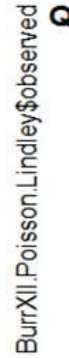
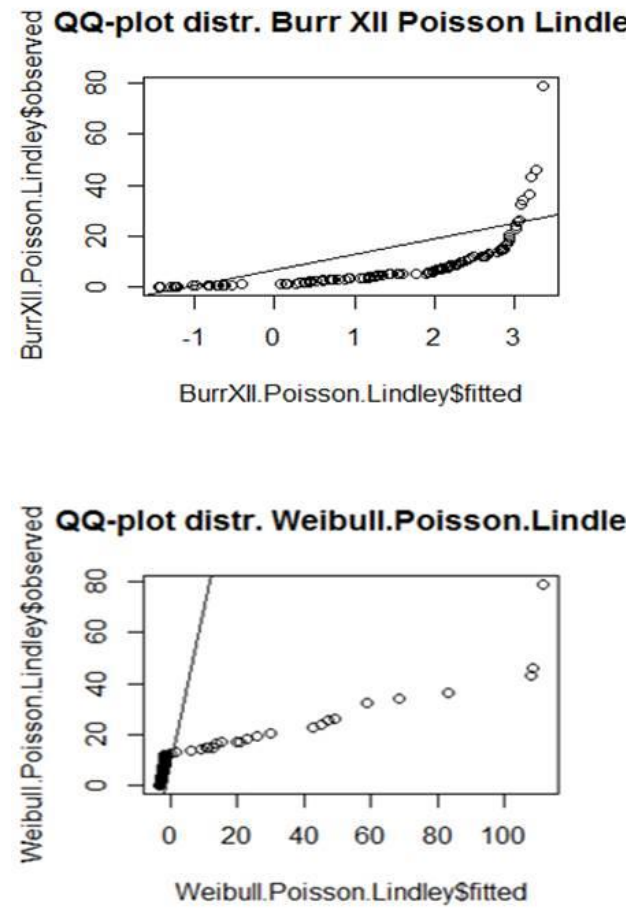
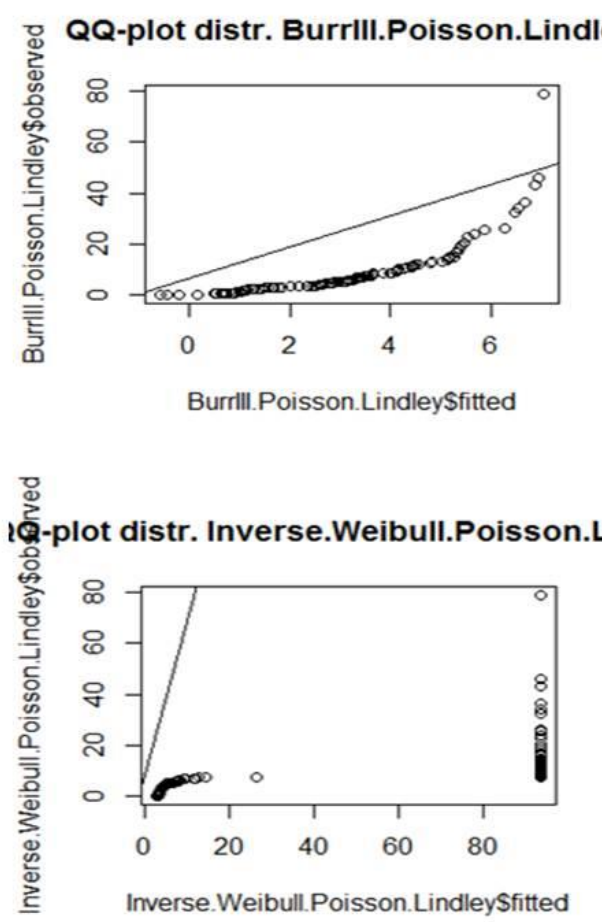

Fig. 9: QQ Plots to Bladder Cancer Patient's Data which Fitted by CBXIIPL, CBIIIPL, CWPL and CIWPL Distributions

\section{Conclusion}

In this article, a new general class of lifetime distributions so called the complementary Poisson Lindley class of distributions is proposed. This new class extends several sub-models. A mathematical treatment of the new class, including expressions for density function, moments and moment generating function are provided. Maximum likelihood inference is implemented for estimating the model parameters. Some sub-model distributions are introduced and fitted to real data set to show the usefulness of the proposed class.

\section{References}

[1] K. Adamidis and S. Loukas, A lifetime distribution with decreasing failure rate, Statistics and Probability Letters, 39, 35-42, 1998. http://dx.doi.org/10.1016/S0167-7152(98)00012-1.

[2] S. Alkarni and A. Oraby, A compound class of Poisson and lifetime distributions, Journal of Statistics Applications and Probability, 1, 45-51, 2012. http://dx.doi.org/10.12785/jsap/010106.

[3] A. Asgharzadeh, H. S. Bakouch, S. Nadarajah and L. Esmaeili, A new family of compound lifetime distributions, Kybernetika, 50, 142-169, 2014. http://dx.doi.org/10.14736/kyb-2014-1-0142.

[4] I. W. Burr, Cumulative frequency functions, Annals of Mathematical Statistics, 13, 215-232, 1942. http://dx.doi.org/10.1214/aoms/1177731607.

[5] I. W. Burr, Parameters for a general system of distributions to match a grid of $\alpha 3$ and $\alpha 4$, Communications in Statistics, 2, 1-21, 1973. http://dx.doi.org/10.1080/03610927308827052.

[6] M. Chahkandi and M. Ganjali, on some lifetime distributions with decreasing failure rate, Computational Statistics and Data Analysis, 53, 4433-4440, 2009. http://dx.doi.org/10.1016/j.csda.2009.06.016.

[7] G. M. Cordeiro and R. B. Silva, The complementary extended Weibull power series class of distributions, Ciência e Natura, 36, 1-13, 2014. http://dx.doi.org/10.5902/2179460X13194.

[8] D. J. Flores, P. Borges, G. Cancho and F. Louzada, The complementary exponential power series distribution, Brazilian Journal of Probability and Statistics, 27, 565-584, 2013. http://dx.doi.org/10.1214/11-BJPS182.

[9] T. C. Headrick, M. D. Pant and Y. Sheng, On simulating univariate and multivariate Burr type III and type XII distributions, Applied Mathematical Sciences, 4(45), 2207 - 2240, 2010.

[10] M. S. Khan, G. R. Pasha and A. H. Pasha, Theoretical analysis of inverse Weibull distribution, WSEAS Transactions on Mathematics, 7, 30$38,2008$.

[11] C. Kus, A new lifetime distribution, Computational Statistics and Data Analysis, 51, 4497-4509, 2007. http://dx.doi.org/10.1016/j.csda.2006.07.017.

[12] A. Leahu, B. G. Munteanu and S. Cataranciuc,S, Max-Erlang and Min-Erlang power series distributions as two new families of lifetime distribution, Buletinul Academiei de Stiinte a Republicii Moldova. Matematica, 2(75): 60-73, 2014.

[13] E. T. Lee and J. W. Wang, Statistical methods for survival data analysis, 3rd edition, Wiley, New York, 2003. http://dx.doi.org/10.1002/0471458546.

[14] S. R. Lindsay, G. R. Wood and R. C. Woollons, Modelling the diameter distribution of forest stands using the Burr distribution, Journal of Applied Statistics, 23(6), 609-619, 1996. http://dx.doi.org/10.1080/02664769623973.

[15] F. Louzada, M. Roman and V. G. Cancho, The complementary exponential geometric distribution: model, properties, and a comparison with its counterpart, Computational Statistics and Data Analysis, 55, 2516-2524, 2011. http://dx.doi.org/10.1016/j.csda.2011.02.018. 
[16] P. R. Tadikamalla, A look at the Burr and related distributions, International Statistical Review, 48, 337-344, 1980. http://dx.doi.org/10.2307/1402945.

[17] R. Tahmasbi and S. Rezaei, A two-parameter lifetime distribution with decreasing failure rate, Computational Statistics and Data Analysis, 52 , 3889-3901, 2008. http://dx.doi.org/10.1016/j.csda.2007.12.002. 